\title{
Venous thromboembolism incidence in mental health services for older people: survey of in-patient units
}

\author{
Martin van Zyl, ${ }^{1}$ Gillian Wieczorek, ${ }^{2}$ Joe Reilly $^{3}$
}

The Psychiatrist (2013), 37, 283-285, doi: 10.1192/pb.bp.112.041848

${ }^{1}$ Bowes Lyon Unit, Durham; ${ }^{2}$ Centre for the Health of the Elderly, Campus for Ageing and Vitality, Newcastle upon Tyne; ${ }^{3}$ Wolfson Research Institute, Stockton on Tees

Correspondence to Martin van Zyl (martin.vanzyl@nhs.net)

First received 24 Oct 2012, accepted 6 Mar 2013
Aims and method To establish the incidence of venous thromboembolism (VTE) in mental health services for older people (MHSOP) in-patient units, and investigate current practice in terms of assessment of risk of VTE and treatment given. We conducted an incidence survey of VTE on in-patient units in MHSOP in a National Health Service trust, searching data of in-patients discharged over a 2-year period.

Results Overall, 1495 individual patient records were searched. There were 17 confirmed VTEs, which is comparable with VTE incidence rates in general hospitals. There were no risk assessments done on admission and no thromboprophylaxis given. Use of compression stockings and mobilisation was limited.

Clinical implications The study confirmed a significant VTE incidence and the need for increased awareness among MHSOP staff. This can be met by improved training of VTE awareness and management for clinical staff and the use of a VTE assessment tool on MHSOP in-patient units.

Declaration of interest None.
The UK population is ageing and the percentage of persons aged 65 and over is increasing; it rose from 15\% in 1985 to $17 \%$ in 2010, an increase of 1.7 million people. It is projected that by 2035 those aged 65 and over will account for $23 \%$ of the total population. ${ }^{1}$ With older age comes a higher risk of deep-vein thrombosis (DVT), which may in turn lead to pulmonary embolism. Venous thromboembolism (VTE), as these two conditions are collectively known, is the third most common cardiovascular disease after myocardial infarction and stroke in medical and surgical patients. ${ }^{2}$ Current failure to adequately screen and prevent VTE is believed to cause annually between 25000 and 32000 potentially avoidable deaths in the UK. ${ }^{3}$ Inconsistent use of prophylactic measures further contributes to the incidence of this condition. ${ }^{4}$ Apart from age, risk factors for VTE include immobility; active cancer or cancer treatment; dehydration; known thrombophilias; obesity; significant medical comorbidities; personal history of VTE or VTE in a first-degree relative; use of hormone replacement therapy or the oral contraceptive pill; and pregnancy. ${ }^{5}$

Incidence of VTE in older patients on general in-patient units has been well documented; the incidence of DVT is estimated to be approximately 655 per 100000 population and of pulmonary embolism 254 per 100000 population. ${ }^{6}$ Less is known, however, about the incidence of VTE in older people using mental health services. A clear association of VTE with antipsychotics has been established. ${ }^{7}$ This increased risk is low in absolute terms (4 additional cases per 10000 exposed patients per year), but appears to be more marked among new users of atypical antipsychotics. ${ }^{8}$
Elderly patients are among the most common recipients of antipsychotics. ${ }^{9}$ Underlying risk factors present in older psychiatric patients may further contribute to the etiopathogenesis of VTE. Such patients often present with the established risk factors for VTE. Additionally, immobilisation due to physical restraint and pharmacotherapy with benzodiazepines and antipsychotics may put older people using mental health services at a higher risk of VTE. $^{10}$

Reviewing the literature revealed limited evidence with regard to older people in psychiatric settings. One multicentre cross-sectional study ${ }^{11}$ conducted in psychiatric in-patients, which included patients using mental health services for older people (MHSOP), used a standardised questionnaire and a systematic compression ultrasound and found a VTE incidence of $6 \%$. Another multicentre study in subacute care departments similar to MHSOP detected DVT in $16 \%$ of patients using systematic ultrasound examination. $^{12}$ One National Health Service (NHS) trust providing mental healthcare investigated mortality and morbidity linked to VTE in the previous year and found five deaths primarily due to VTE (two in MHSOP, two in substance misuse services and one in general adult mental health services). ${ }^{13}$ A retrospective study on nursing homes investigating the effects of antipsychotics found a hospitalisation rate for VTE of 0.91 per 100 person years. ${ }^{9} \mathrm{~A}$ recent incidence study using a national database for nursing home residents yielded an incidence of 3.68 cases per 100 person years. $^{14}$ 
However, rigorous scientific data such as randomised controlled trials for risk/benefit ratio and efficacy of prophylactic treatment are lacking in this patient group. The benefit of thromboprophylactic treatment needs to be weighed up against the risks of adverse effects such as bleeding and drug interactions, especially in older people.

As there is very little good-quality published evidence on the incidence of VTE in psychiatric in-patients, we conducted an incidence survey of VTE on in-patient units in MHSOP in Tees, Esk and Wear Valleys NHS Foundation Trust. The survey was approved by the trust MHSOP quality and assurance group and registered as clinical research. We were advised that ethical approval was not needed.

\section{Method}

We searched patient data from the electronic patient record system used in the trust called Paris. The Paris system was first implemented in 2008 and contains all records of MHSOP in the trust since then. To assess sufficient data, we searched the records of all patients discharged from MHSOP in-patient wards throughout the trust over a 24-month period (July 2009-June 2011). This included all discharges from MHSOP in-patient units in North Durham, South Durham, North Tees, South Tees and North Yorkshire. In all, we searched the data of 1495 patient discharges.

The Paris system has the capacity to create a summary of an individual patient's case notes. We were therefore able to conduct a word search of the 'case-note summary' on every patient's record throughout their admission. To identify all VTEs, we performed our search in two stages:

- stage 1 , to identify all confirmed VTEs and pulmonary embolisms, we performed keyword searches using: 'VTE', 'DVT', 'PE', 'pulm', 'embol' and 'thromb';

- stage 2, having identified a VTE or pulmonary embolism, we then carefully searched all case notes recording the following information for each patient with VTE: age

gender

psychiatric diagnosis

whether a DVT or pulmonary embolism was diagnosed treatment given

whether a VTE risk assessment was completed on admission

outcome following treatment

all comorbidities

antipsychotic exposure.

\section{Results}

By searching the 1495 individual patient records, we found 17 confirmed cases of VTE: 12 were DVTs (72\%) and 5 pulmonary embolisms (28\%). The subsequent incidence was estimated at 1.14 VTE cases per 100 patients discharged.

The mean age of the patients diagnosed with VTE was 78 years (range 60-89). There were more females with VTEs, as would be expected, with the male/female ratio of $29 \%$ to $71 \%$.
We found that the most common diagnoses of patients with VTE were depression (29\%) and vascular dementia (23\%). Other diagnoses included schizophrenia (12\%), organic psychosis (12\%), Alzheimer's dementia (6\%), bipolar disorder $(6 \%)$, frontotemporal dementia (6\%) and youngonset dementia (6\%). Most patients with VTE (76\%) were prescribed antipsychotics, most commonly olanzapine (60\%). The next most commonly prescribed antipsychotics were risperidone and quetiapine (both $12 \%$ ).

Examining comorbidities revealed that, apart from age, $47 \%$ of patients did not have clear risk factors for VTE. However, malignancy (24\%), chronic physical disease (18\%) and immobility (12\%) were present in a significant number of patients.

In terms of VTE risk assessment and preventive measures (thromboprophylaxis and mobilisation), there was very little to be found, with no record of patients with VTE having received a VTE risk assessment or thromboprophylaxis. Only 29\% of patients' records had clear evidence that mobilisation was encouraged.

Finally, we also examined the treatment given following diagnosis of VTE and the outcome resulting from that. We found that all patients were treated appropriately with low molecular weight heparin (LMWH) and warfarin, however, the use of compression stockings was limited, with only 5 of the 12 patients diagnosed with DVT receiving a compression stocking. In terms of outcome, $35 \%$ of patients (all diagnosed with pulmonary embolism) were transferred to an acute setting and there were no deaths recorded from the 17 patients with VTE.

\section{Discussion}

This study's findings add to a neglected area of research. The incidence of VTE in our study (1.14 VTE cases per 100 patients discharged) was comparable with incidence data from acute trusts. ${ }^{6}$ We believe this highlights the need for increased awareness of VTE among staff working in MHSOP.

Despite the significant incidence documented in this study, no patient had a recorded VTE risk assessment on admission. To improve early identification of patients who may benefit from thromboprophylaxis, a Department of Health VTE assessment tool ${ }^{15}$ will be piloted on one MHSOP in-patient ward in our trust with a view to wider use in future. Although we recognise the importance of identifying older patients in mental health settings at risk for VTE, we acknowledge the importance of future research with regard to the risk and benefit of prophylactic treatment for VTE in this patient group.

In our study, patients were appropriately treated with LMWH and warfarin, but the use of compression stockings was limited. There was also limited evidence of mobilisation being considered as part of VTE management (29\%). These two issues could be addressed with better training of MHSOP clinical staff via mandatory training and e-learning tools. The Department of Health has provided an e-learning tool (www.e-lfh.org.uk/projects/vte/index.html) which, as a result of our findings, will be distributed to our clinical staff.

In our study the majority of the patients diagnosed with VTE were taking antipsychotics at the time. The size of 
study limits inferences, however, our findings do support evidence of an association between atypical antipsychotics and VTE.

Considering the limitations of our study, the Paris database only has a limited search capacity and we may not have accounted for all cases of VTE as not all case records may have been recorded on Paris. However, an underestimation of VTE incidence should not negate the conclusions of our survey. We did not examine critical incident data, so patients dying on in-patient units as a result of VTE would not be included in our data. Similarly, mortality data from patients who may have died after being transferred to an acute setting were not examined.

\section{Acknowledgements}

We thank our trust colleagues Andrew Ntanda, Corrie Burton and Lesley Chapman who helped with the Paris search.

\section{About the authors}

Martin van Zyl is ST5 in old age psychiatry, Bowes Lyon Unit, Durham, Tees, Esk and Wear Valleys NHS Foundation Trust; Gillian Wieczorek is ST5 in old age psychiatry, Centre for the Health of the Elderly, Campus for Ageing and Vitality, Newcastle upon Tyne, Northumberland, Tyne and Wear NHS Foundation Trust; Joe Reilly is professor of mental health, Wolfson Research Institute, Stockton on Tees, Tees, Esk and Wear Valleys NHS Foundation Trust.

\section{References}

1 Office for National Statistics. Population Ageing in the United Kingdom, its Constituent Countries and the European Union. ONS, 2012.

2 Giuntini C, Ricco GD, Marini C, Mellilo E, Palla A. Pulmonary embolism: epidemiology. Chest 1995; 107 (suppl 1): 3S-9S.
3 Roopen A (ed.). Venous Thromboembolism Prevention: A Patient Safety Priority. King's Thrombosis Centre, 2009.

4 Hunt BJ. Awareness and politics of venous thromboembolism in the United Kingdom. Arteriosclerotic Thromb Vasc Biol 2008; 28: 398-9.

5 National Institute for Health and Clinical Excellence. Venous Thromboembolism: Reducing the Risk. Reducing the Risk of Venous Thromboembolism (Deep Vein Thrombosis and Pulmonary Embolism) in Patients Admitted to Hospital (Clinical Guideline CG92). NICE, 2010.

6 Stein PD, Hull RD, Kayali F, Ghali WA, Alshab AK, Olson RE. Venous thromboembolism according to age: the impact of an aging population. Arch Intern Med 2004; 164: 2260-5.

7 Hägg S, Jönsson AK, Spigset O. Risk of venous thromboembolism due to antipsychotic therapy. Expert Opin Drug Saf 2009; 8: 537-47.

8 Parker C, Coupland C, Hippisley-Cox J. Antipsychotic drugs and risk of venous thromboembolism: nested case-control study. BMJ 2010; 341 4245.

9 Liperoti R, Pedone C, Lapane K, Mor V, Bernabei R, Gambassi G. Venous thromboembolism among elderly patients treated with atypical and conventional antipsychotic agents. Arch Int Med 2005; 165: 2677-82.

10 Mal R, Masopust J, Hosák L, Konupcíková K. Assessment of risk of venous thromboembolism and its possible prevention in psychiatric patients. Psychiatry Clin Neurosci 2008; 62: 3-8

11 Laeut K, Dellue A. Incidence of venous thromboembolism in psychiatric units. J Thrombosis Haemostasis, July 2011, vol./is. 9/(607-608), 15387933.

12 Bosson JL, Labarere J, Sevestre MA, Belmin J, Beyssier L, Elias A, et al. Deep vein thrombosis in elderly patients hospitalized in subacute care facilities: a multicenter cross-sectional study of risk factors, prophylaxis, and prevalence. Arch Intern Med 2003; 163: 2613-8.

13 Aitken PHL. Venous thromboembolism is a preventable cause of death in people using mental health services. BMJ 2009; 8 Dec.

14 Reardon G, Pandya N, Nutescu EA, Lamori J, Damaraju CV, Schein J, et al. Incidence of venous thromboembolism in nursing home residents. J Am Geriatr Soc 2012; 60 (S150): 0002-8614.

15 VTE Prevention NHS England. Risk Assessment for Venous Thromboembolism (VTE). VTE Prevention NHS England, 2010. 